\title{
Juvenile psammomatoid Ossifying Fibroma
}

\author{
Dr. Swapnil Moghe ${ }^{1}$, Dr. Anjali Moghe ${ }^{2}$, Dr. Ajay Pillai ${ }^{3}$, Dr. Saksham Nahar ${ }^{4}$, \\ Dr. Neha Goyal ${ }^{5}$, Dr. M.D. Khan ${ }^{6}$ \\ ${ }^{1}$ (Reader, Department Of Oral \& Maxillofacial Surgery, Peoples Dental Academy, Peoples University, Bhopal, India.) \\ ${ }^{2}$ (Consultant Dental \& Cosmetic Surgeon, Bhopal Care Hospital, Bhopal, India.) \\ ${ }^{3}$ (Reader, Department Of Oral \& Maxillofacial Surgery, Peoples Dental Academy, Peoples University, Bhopal, India) \\ ${ }^{4}$ (PG Student, Department Of Oral \& Maxillofacial Surgery, Peoples Dental Academy, Bhopal, India) \\ 5 (Consultant Dental Surgeon, Govt. District hospital, Mungeli.) \\ ${ }^{6}$ (Lecturer, Department Of Oral \& Maxillofacial Surgery, Peoples Dental Academy, Bhopal, India)
}

\begin{abstract}
Ossifying fibromas are uncommon benign lesion with aggressive local growth. These tumors thought to originate from the periodontal ligament. WHO 2005 classified juvenile ossifying fibroma (JOF) into juvenile psammomatoid ossifying fibroma and juvenile trabecular ossifying fibroma. These tumors must be treated by radical excision to prevent recurrence. We report a case of JOF of psammomatoid pattern in a 12 year old boy, the treatment was radical en bloc resection.
\end{abstract}

Keywords: Ossifying Fibroma, Juvenile ossifying fibroma, Psammomatoid ossifying fibroma

\section{Introduction:}

Benign fibro-osseous lesion of head and neck region are uncommon and constitute a wide range of Juvenile Ossifying Fibroma is often difficult to distinguish between other Fibro Osseous Lesions As it has been stated that JOF predominantly found in the mandible. It has also been reported in frontal, ethmoidal, Sphenoidal and temporal bones as well as the orbit and anterior cranial fossa. The author report a case of psammomatoid JOF of maxilla which is found to be rare pathology. It is generally seen in younger age group. The trabecular variant of juvenile ossifying fibroma was previously alluded to by Reed and Hagy, in 1965.

Case Presentation:

A twelve-year-old systemically healthy boy, presented with swelling on the left mid face since two years.Perceived 5 years before consultation, swelling was as small as peanut sized growth and gradually increased in size slowly.Swelling was diffused and asymptomatic. Intra oral swelling extends from anteriorly from left anterior maxilla to posteriorly to the region of left first molar and superiorly from upper buccal vestibule, extending inferiorly to the level of attached gingiva. The buccal cortical plate was expanded with no mucosal changes. The swelling was hard in consistency with no fluctuation of approximately size of $2.5 \times 3 \times 2$ $\mathrm{cm}$. Physical examination revealed no deformity of the orbital region and confirmed no nasal obstruction by a mass lined by normal mucosa.

On clinical examination, a mild diffused swelling (Figure 1) over the left mid facial region, with slight facial asymmetry was observed. On palpation, swelling was firm with no associated tenderness or raise in temperature. Intra orally, a solitary swelling, covered by normal mucosa, about $4 \times 3 \mathrm{cms}$, oval in shape was seen extending from 21 to 26 (Figure 2). Palpation revealed,Swelling was multi lobular, hard in consistency and slight obliteration of the buccal vestibule. Testing of dental vitality proved positive for upper left 21-27.No lymphadenopathy was detected and mouth opening was normal. Under G.A., a crevicular incision was placed from $21-27 \&$ a full thickness mucoperiosteal flap was raised. The site was exposed $\&$ the encapsulated mass was removed in Toto. (Figure 3).The lesion site was irrigated with betadine \& saline. Haemostasis was achieved. Closure was done with 3-0 vicryl. Patient was extubated \& postoperative recovery was uneventful. 


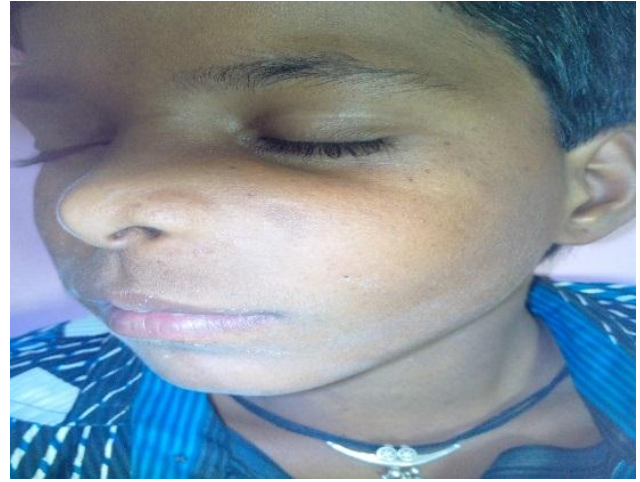

Fig 1: Diffuse swelling over midface.

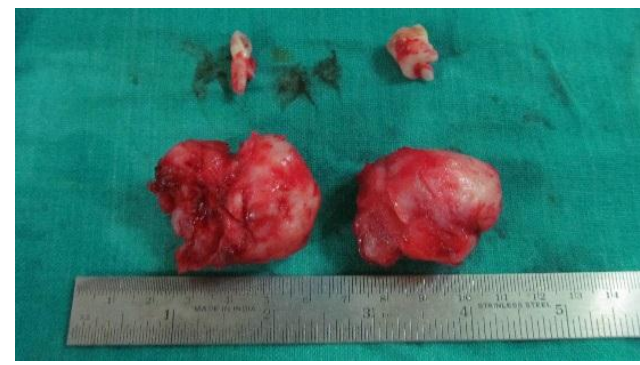

Fig 3: Excised tumor mass in Toto.

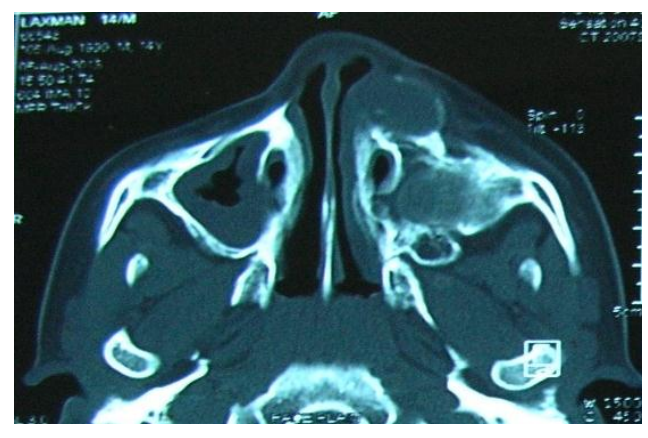

Fig 5: CT scan showing involvement of Left Maxillary antrum.

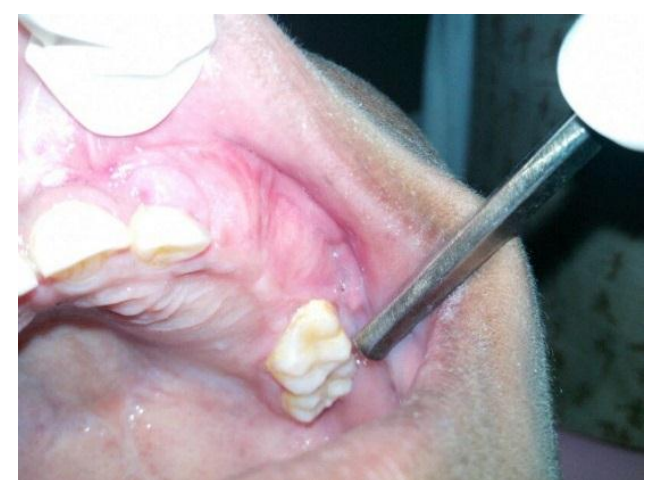

Fig 2: Intraoral vestibule obliteration from 21-26.

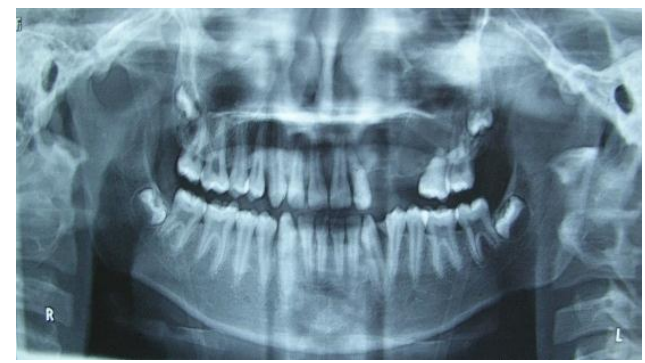

Fig 4: OPG Showing displacement of 22

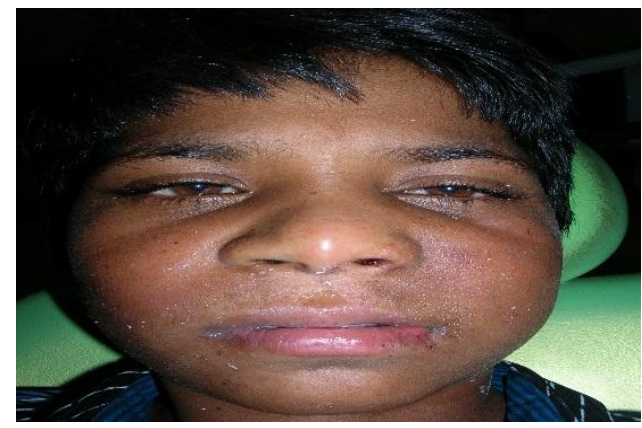

Fig 6: Post operative profile view after 1 month.

\section{Investigation}

1. An intraoral periapical radiograph revealed a mixed radiolucent- radiopaque image with missing 23-25.

2. The orthopantomography examination revealed an image showing displacement of teeth 22 , with an abnormal degree of opacity at the dental roots (Figure 4). The centre of the lesion appeared radiolucent with flecks of radiopaque foci, while the margins were more radiopaque. The lesion extended from the bone crest to approximately $3 \mathrm{~cm}$ above apical level of the adjacent teeth.

3. Computed tomography of the facial bones was advised which demonstrated a relatively well circumscribed lesion on the left maxilla with no involvement of the nasal septum and left orbital floor (Figure 5). This heterogeneous tumour was of measuring $2.5 \times 3 \times 2 \mathrm{~cm}$.

4. Aspiration yielded negative results, ruling out any cystic lesion.

5. The incisional biopsy was performed under local anaesthesia. Histopathology report which revealed tumour composed of spindle fibroblastic cells and osteoblast cells were present. And along with clinicoradiographic findings, reported it as ossifying fibroma. 


\section{Differential Diagnosis}

With a condition evolving slowly, painlessly, and with no related neurological symptoms, the diagnosis should look toward a benign neoplastic process. Ossifying fibroma, Pindborgtumor, the odontogenicadenomatoid tumor, cemento-ossifying fibroma. The mixed odontogenic tumors, odontoma and ameloblastic fibro-odontoma. Peripheral giant cell granuloma ${ }^{1}$ also occur in younger patients and present as mixed density mass lesions in the tooth bearing portions of the jaws.

\section{Outcome And Follow-Up}

The postoperative course was uneventful and there were no signs of recurrence after a periodic followup of 1 month. Follow up of 1 month (Figure 6) revealed substantial healing of bony defect.

\section{Discussion}

Ossifying fibroma is an uncommon benign osteogenic neoplasm, accounting for $2 \%$ of oral tumors in children ${ }^{2}$. Juvenile ossifying fibroma behaves aggressively locally and has a high recurrence rate when not treated adequately, the correct treatment being en bloc resection with free surgical margins. The juvenile form could be distinguished from ossifying fibroma by the following features: earlier onset (at childhood or adolescence), locally aggressive growth and osteoid trabeculae on histological examination ${ }^{2}$.

Regezzi and Sciubba ${ }^{3}$ stated that multiple Ossifying fibroma are sporadic, but there is familial inclination in few cases. They also stated that chromosomal translocations were seen in few instances.

The clinical evolution of the tumor usually is as follows: Initially asymptomatic, the tumor reaches a point causing a painless swelling of the involved bone as well as functional alterations and cosmetic deformities ${ }^{4}$.Displacement of the teeth may be seen but the teeth remain vital and the overlying mucosa is characteristically intact, although most lesions are discovered during routine dental examinations. Centrifugal growth of the tumor usually causes bowing of the inferior border of the mandible but cortical perforation is rare. We noticed cortical perforation in our case, which is a rare phenomenon as reported in literature.

In Fibro- osseous lesions, normal bone is replaced by fibroblasts and collagen fibers, containing variable amounts of mineralized material. This group comprises of fibrous dysplasia, benign fibro-osseous neoplasms (central ossifying fibroma), and a heterogeneous reactive lesions (osseous dysplasias) ${ }^{5}$.

Juvenile ossifying fibroma are more radiopaque than conventional lesions and they have a 'ground glass' appearance or may form dense lobulated masses ${ }^{6}$.

Radiologically, juvenile ossifying fibroma presents as a clearly circumscribed, concentrically expanding, solitary mass with bone density. It is the circumscribed nature of ossifying fibroma which distinguishes it from fibrous dysplasia.

Histologically, these lesions are always benign, composed of highly vascular and fibroblast-rich connective tissue, which produces a calcified substance that often cannot be clearly attributed to either cement or bone. Clumps of osteoblasts are also present. The incidence of juvenile ossifying fibroma is unknown. A literature review revealed 17 cases reported between 2003 and 2010 with a sex-ratio of five females for one male in adults, while a male predominance is observed in the juvenile form ${ }^{7}$.

Complications of lesions involving the paranasal sinuses includes ocular disturbances, intracranial extension, cysts, recurrences, and, rarely, aggressive growth in the younger age group. The management of these lesions is directed at surgical intervention when complications have occurred, to prevent complications, or to prevent recurrences and must consist of complete surgical excision. Partial resection has been shown to be associated with recurrence ${ }^{8}$.

Desmoplastic fibroma of the maxillofacial region may be difficult to diagnose early in the disease process because of a slow, insidious onset coupled with unremarkable radiographic findings. It is a disease of young people, with a peak incidence in the second decade of life ${ }^{9}$.

\section{References}

[1]. Moghe S etal, Peripheral Giant Cell Granuloma: A Case Report and Review of Literature.People's Journal of Scientific Research Vol. 6(2), July 2013.

[2]. Paulo Roberto Domingueteetal,Juvenile ossifying fibroma of the jaw, BJOMS 46 (2008), $480-81$.

[3]. Regezzi JA, Sciubba JJ. Oral pathology. 3rd ed. Philadelphia: W.B. Saunders; 1999. p. 357-60.

[4]. Martín-Granizo R, Sánchez-Cuellar A, Falahat F. Cemento ossifying fibroma of the upper gingivae. Otolaryngol Head Neck Surg 2000; $122: 775 . s$

[5]. S.M. Gondivkar et al. / Oral Oncology 47 (2011) 804-809

[6]. Paul M. Speight etal, Maxillofacial fibro-osseous lesions, Current Diagnostic Pathology (2006) 12, 1-10.

[7]. R. Breheretetal, Juvenile ossifying fibroma of the maxilla, European Annals of Otorhinolaryngology, Head and Neck diseases (2011) 128, 317-320.

[8]. Abhay M. Vaidya etal,Juvenile aggressive ossifying fibroma presenting as an ethmoid sinus mucocele. Otolaryngol Head Neck Surg 1998;119: 665-8.

[9]. K.N. Guru etal, Desmoplastic Fibroma of the Maxilla - A Rare Case Report. People's Journal of Scientific Research,Vol. 6(1),Jan. 2013. 